\title{
ADEQUAÇÃO DAS PROPRIEDADES MECÂNICAS DE PERFIS ASTM A572 G60 PARA PRODUÇÃO DE TORRES DE TRANSMISSÃO*
}

Izabelle Lannes Salgueiro Ferreira' Gabriela Martins Lazarotto llaciara Medeiros de Freitas ${ }^{3}$

\section{Resumo}

O presente trabalho teve como objetivo adequar às propriedades mecânicas dos perfis ASTM A572 G60, através do estudo da influência dos parâmetros de processo de laminação e composição química do aço. Os perfis ASTM A572 G60, são utilizados nas indústrias de fabricação de torres de transmissão. A metodologia utilizada neste trabalho foi o desenvolvimento de um modelamento matemático, com base na ferramenta estatística de Análise de Regressão Múltipla. Através desta ferramenta foi possível obter previsibilidade de processo e identificar os parâmetros mais influentes nas propriedades mecânicas dos perfis ASTM A572 G60, o que possibilitou a determinação da composição química ideal do aço e os melhores parâmetros de processo de laminação.

Palavras-chave: Propriedade mecânica; ASTM A572 G60; Parâmetros de processo de laminação; Análise de regressão múltipla.

\section{MECHANICAL CHARACTERISTICS ADEQUATION OFASTM A572 G60 PROFILES FOR APPLICATION IN THE TRANSMISSION TOWERS PRODUCTION}

\section{Abstract}

The present study aimed to suit the mechanical properties of ASTM A572 G60 profiles through the study of the influence of rolling process parameters and steel chemical composition. The ASTM A572 G60 profiles are used in the manufacturing of transmission towers industries. The methodology used in this work (PDCA) was the development of a mathematical model, based on the statistical tool of Multiple Regression Analysis. With this tool, it was possible to obtain predictability of process and to identify the most influential parameters on the mechanical properties of ASTM A572 G60 profiles, which allowed the determination of optimal chemical composition of steel and the best parameters of the rolling process.

Keywords: Mechanical properties; ASTM A572 G60; Rolling process parameters; Multiple regression analysis.

1 Engenharia Metalúrgica UFF, Engenheira de Processo, Gerencia de Laminação a Quente, Votorantim Siderurgia, Barra Mansa, Rio de Janeiro, Brasil.

2 Engenharia Química UFPR, Engenheira de Processo, Gerencia de Produto e Processo, Votorantim Siderurgia, Barra Mansa, Rio de Janeiro, Brasil.

3 Engenharia Metalúrgica UFF, Engenheira de Produto, Gerencia de Produto e Processo, Votorantim Siderurgia, Barra Mansa, Rio de Janeiro, Brasil. 


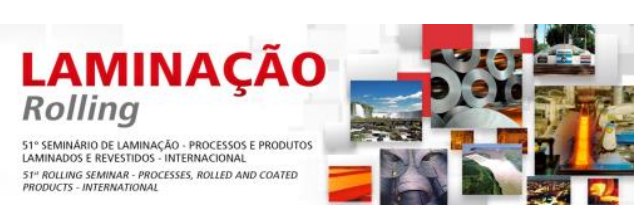

\section{INTRODUÇÃO}

No Brasil, as principais razões para a utilização de torres de aço são a crescente demanda do sistema de telecomunicação e a expansão das linhas de transmissão de energia. Visando atender este atraente mercado de torres, a Votorantim Siderurgia desenvolveu uma linha de perfis de acordo com a Norma ASTM A572 G50 e G60 (2007) [1].

O desenvolvimento deste trabalho se fez necessário devido à dificuldade em atender as propriedades mecânicas dos perfis ASTM A572 G60.

Através da análise da capabilidade de processo, foi possível identificar $17 \%$ de probabilidade de não atendimento dos resultados dos limites de escoamento dos perfis $\mathrm{G} 60$.

O presente trabalho teve por objetivo adequar às propriedades mecânicas dos perfis ASTM A572 G60. A metodologia utilizada foi o PDCA que possibilitou o desenvolvimento de um modelamento matemático, o qual correlaciona através da ferramenta estatística de Análise de Regressão Múltipla, a composição química do aço e os parâmetros de processo de laminação, para obter as propriedades mecânicas do produto.

A norma de referência utilizada neste trabalho para fabricação de perfis foia ASTM A572 G50 e G60 (2007), que permite a utilização de nióbio (Nb) e/ou vanádio (V) nos aços a fim de se obter as características mecânicas adequadas.

\section{MATERIAIS E MÉTODOS}

Para o desenvolvimento deste projeto inicialmente foram coletados dados das propriedades mecânicas do material de interesse, pelo período de um ano e calculada a capabilidade do processo, a qual indicou uma probabilidade de não atendimento à norma em 17\%, com um nível sigma de 2,45.

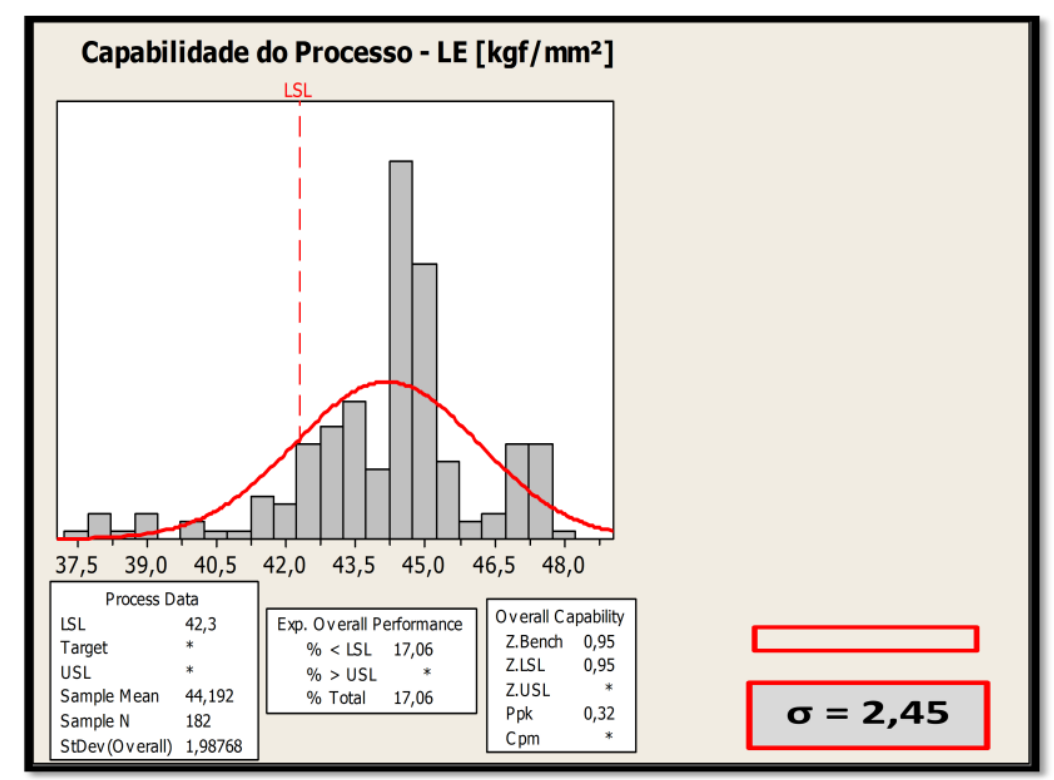

Figura 1 - Teste de capabilidade do processo para o limite de escoamento das cantoneiras ASTM A572 G60.

A metodologia que se aplica para o nível sigma encontrado é o PDCA. Este método é simples, eficaz e composto por quatro etapas:

* Contribuição técnica ao $51^{\circ}$ Seminário de Laminação - Processos e Produtos Laminados e Revestidos, 28 a 31 de outubro de 2014, Foz do Iguaçu, PR, Brasil. 
1a) P (plan - planejamento): Identificar o problema, analisar as causas fundamentais e realizar o plano de ação;

$\left.2^{\text {a }}\right)$ D (do - fazer): Executar as ações planejadas;

$3^{\text {a) }} \mathrm{C}$ (check): Verificar a efetividade das ações;

4ª) A (action): Padronização e conclusão

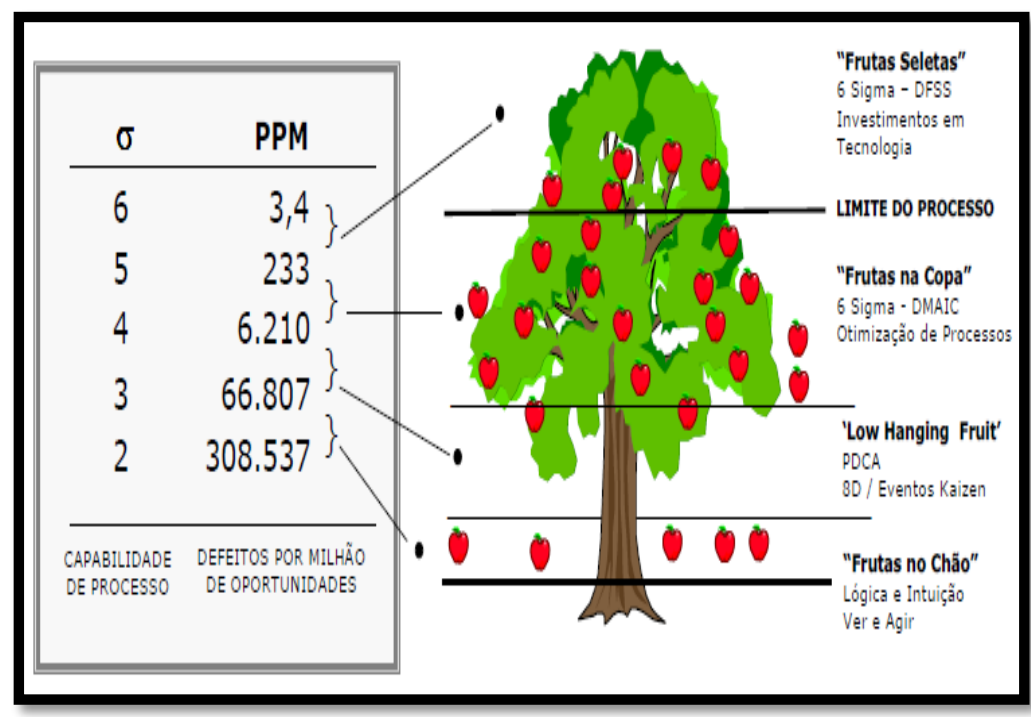

Figura 2 - Árvore de análise de nível sigma versus Metodologia adequada de projeto.

Durante a etapa do planejamento, foi realizado um documento de coleta de dados para identificar e registrar os parâmetros de processo do laminador (temperatura do forno, velocidade de laminação, percentual de redução, etc.).

\begin{tabular}{|c|c|c|c|c|c|c|c|c|c|}
\hline \multicolumn{10}{|c|}{ REGISTRO DE PARÂMETROS DE PROCESSO } \\
\hline Obs: & \multicolumn{9}{|c|}{ Laminador Contínuo de Perfis - Teste Composição Química Cant 100} \\
\hline Material & Quantidade(t) & № de peças & Corrida & $\begin{array}{c}\text { Início de } \\
\text { enfornamento }\end{array}$ & \begin{tabular}{|c}
$\begin{array}{c}\text { Horário de saída do } \\
\text { forno (12barra) }\end{array}$ \\
\end{tabular} & \begin{tabular}{|c|}
$\begin{array}{c}\text { Velocidade } \\
(\mathrm{m} / \mathrm{s})\end{array}$ \\
\end{tabular} & $\operatorname{GAP}(s)$ & \begin{tabular}{|c|}
$\begin{array}{c}\text { Curva de } \\
\text { aquecimento }\end{array}$ \\
\end{tabular} & $\begin{array}{c}\text { Temperatura } \\
\text { Zonas } 7 \text { e } 8 \\
\end{array}$ \\
\hline \multirow{2}{*}{$\begin{array}{c}\text { Cant } 100 \times 7 \\
\text { G60 }\end{array}$} & $16 t$ & 9 tarugos & 4000017384 & 24:02:00 & $2: 14: 00$ & 3,53 & 15 & 2 & 1182 \\
\hline & & & & & & & & & 1200 \\
\hline \multicolumn{3}{|c|}{ Temperatura tarugo saída do forno } & \multicolumn{3}{|c|}{ Temperatura tarugo na $\mathrm{G} 2$} & Temperatu & Tempera & Tempo de água & \\
\hline Cabeça & Meio & Cauda & Cabeça & Meio & Cauda & ra G17 & tura leito & (nuvem meio & Obs \\
\hline 990 & 1020 & 995 & 890 & 900 & 890 & 950 & $*$ & \multirow{5}{*}{12} & * Não foi possível \\
\hline 987 & 1010 & 990 & 887 & 890 & 890 & 953 & $*$ & & identificar a T no \\
\hline 995 & 1014 & 987 & 880 & 877 & 870 & 954 & $*$ & & leito, \\
\hline 990 & 1012 & 995 & 904 & 894 & 892 & 949 & 855 & & \\
\hline 985 & 1010 & 986 & 892 & 880 & 861 & 950 & 849 & & \\
\hline
\end{tabular}

Figura 3 - Exemplo da tabela de registro de parâmetros de processo de laminação.

O aço utilizado para fabricação dosperfis A572 G60 era o 1520-B, com custo médio de liga de 87,97R \$/t, com a seguinte composição química: 


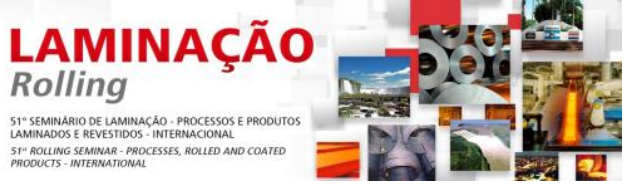

Tabela 1 - Tabela de composição química do aço 1520-B.

\begin{tabular}{|c|c|c|c|c|c|c|c|c|c|c|c|c|c|}
\hline \multirow{2}{*}{ Material } & \multicolumn{13}{|c|}{ Composição Química (\%) } \\
\hline & C & $\mathrm{Mn}$ & $\mathrm{Si}$ & $\mathbf{P}$ & $S$ & $\mathrm{Cu}$ & Sn & $\mathrm{Ni}$ & $\mathrm{Cr}$ & Mo & $\mathrm{Nb}$ & $\mathrm{N}$ & B \\
\hline \multirow{2}{*}{$\begin{array}{c}\text { 1520-B A572 } \\
\text { G60 }\end{array}$} & 0,17 & 1,49 & 0,20 & - & - & - & - & - & - & - & 0,015 & - & - \\
\hline & 0,22 & 1,60 & 0,30 & 0,04 & 0,05 & 0,35 & 0,10 & 0,30 & 0,20 & 0,10 & 0,030 & 125,00 & - \\
\hline
\end{tabular}

Existem duas maneiras de se obter propriedades mecânicas nos metais e ligas metálicas, adequação da composição química ou tratamentos térmicos/termomecânicos [2-4].

Analisando os parâmetros de processo do laminador e a composição química do aço 1520-B, o primeiro experimento proposto foi utilizar o refinador de grão, $\mathrm{Nb}$, no teor máximo permitido pela norma $(\mathrm{Nb}(\%)=0,030)$.

Após a realização do primeiro experimento, a análise de capabilidade do processo identificou $22 \%$ de probabilidade de não atendimento das propriedades mecânicas especificadas na Norma ASTM A572 G60 e o custo médio da liga aumento de 87,97 $\mathrm{R} \$ / \mathrm{t}$ para $98,89 \mathrm{R} \$ / \mathrm{t}$.

Com base neste primeiro resultado, se fez necessário o estudo aprofundado do $\mathrm{Nb}$ em solução sólida, onde foi possível identificar que quanto maior o teor de $\mathrm{Nb}$ disponível na liga, maior é a temperatura necessária para garantir a solubilização deste elemento.

Conforme a tabela 2 a seguir, observa-se que para o teor de $0,015 \% \mathrm{Nb}$, a uma temperatura de $1130^{\circ} \mathrm{C}, 51,26 \%$ de $\mathrm{Nb}$ encontram-se me solução sólida, porém para $0,030 \% \mathrm{Nb}$, a temperatura necessária aumenta para $1259^{\circ} \mathrm{C}$ e o percentual de $\mathrm{Nb}$ em solução sólida cai para $20 \%$, ou seja, apenas $20 \%$ do $\mathrm{Nb}$ adicionado na liga é eficaz como refinador de grão conferindo propriedade mecânica ao material [6-7].

Tabela 2 - Tabela de simulação de aços longos microligados ao Nióbio.

Technical Metallurgical Analysis for long products Microalloyed Nb

\begin{tabular}{|c|c|c|c|}
\hline Product & \multicolumn{3}{|c|}{1520} \\
\hline Thickeness $\mathrm{mm}$ & 10 & 10 & 10 \\
\hline Reheat temperature $\mathrm{C}$ & 1050 & 1050 & 1050 \\
\hline Last Pass Temeperature C & 870 & 870 & 870 \\
\hline$\% \mathrm{C}$ & 0,2 & 0,17 & 0,22 \\
\hline$\% \mathrm{Mn}$ & 1,45 & 1,45 & 1,45 \\
\hline$\% \mathrm{Cu}$ & 0,35 & 0,35 & 0,35 \\
\hline$\% \mathrm{Ni}$ & 0,3 & 0,3 & 0,3 \\
\hline$\% \mathrm{~V}$ & 0 & 0 & 0 \\
\hline$\% \mathbf{N b}$ & 0,015 & 0,015 & 0,03 \\
\hline$\% \mathrm{Al}$ & 0 & 0 & 0 \\
\hline$\% \mathrm{Cr}$ & 0,2 & 0,2 & 0,2 \\
\hline$\% \mathrm{Mo}$ & 0,1 & 0,1 & 0,1 \\
\hline \multicolumn{4}{|c|}{ RESULTS } \\
\hline$\% \mathrm{CE}-(\mathrm{Pcm})$ & 0 & 0,29 & 0,34 \\
\hline $\begin{array}{l}\text { Temperature Recomended for Reheat to keep } \\
100 \% \mathrm{Nb} \text { in Soluction } \mathrm{f}(\mathrm{C}, \mathrm{Nb}, \mathrm{N})\end{array}$ & 1149 & 1130 & 1259 \\
\hline $\begin{array}{l}\% \mathrm{Nb} \text { in Soluction for the aplied Temperature } \\
\text { Reheat }\end{array}$ & 0,007 & 0,008 & 0,006 \\
\hline $\begin{array}{l}\text { Effective \% Nb in soluction for the aplied } \\
\text { Temperature Reheat }\end{array}$ & 0,007 & 0,008 & 0,006 \\
\hline Factor $\mathrm{Nb}$ in Soluction & $43,960 \%$ & $51,26 \%$ & $20,075 \%$ \\
\hline Effective $\mathrm{N} \% \mathrm{f}(\% \mathrm{Al})$ & 0,0125 & 0,0125 & 0,0125 \\
\hline Bainite Start-Bodnar & 613 & 631 & 601 \\
\hline Bainite Start-Kirkaldy & 563 & 565 & 562 \\
\hline
\end{tabular}

* Contribuição técnica ao $51^{\circ}$ Seminário de Laminação - Processos e Produtos Laminados e Revestidos, 28 a 31 de outubro de 2014, Foz do Iguaçu, PR, Brasil. 


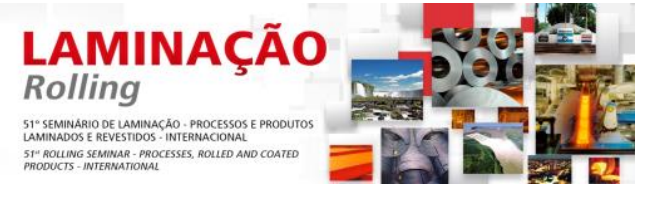

O segundo experimento realizado foi adição de vanádio (V) ao aço 1520-B, tal decisão foi tomada baseada em dois princípios, primeiro que a norma ASTM A572 permite a utilização do $\mathrm{V}$ como refinador de grãos e como segundo pela limitação da temperatura de operação do forno de reaquecimento de $1200^{\circ} \mathrm{C}$, neste caso, é sabido que o $\mathrm{V}$ solubilizar a aproximadamente $800^{\circ} \mathrm{C}$, tornando-se adequado para a obtenção de propriedade mecânica do material. Neste experimento obtivemos $100 \%$ de atendimento as especificações da norma.

De acordo com a nova composição química o segundo experimento foi realizado, através da utilização do software estatístico Minitab um teste de correlação de melhor subgrupo de variáveis, que se destina a identificar as variáveis de entrada mais influentes na variável resposta (propriedade mecânica dos perfis).

Conforme a tabela 3, pode-se verificar que os parâmetros de processo mais influentes foram: temperatura do tarugo na saída do forno de reaquecimento ( $\left.T_{G 2}\right)$, percentual de $\mathrm{Nb}, \mathrm{V}$ e $\mathrm{Mn}$.

Tabela 3 - Tabela resposta da análise de melhor subgrupo para determinar a variável resposta.

\begin{tabular}{|c|c|c|c|c|c|c|c|c|c|}
\hline Vars & R-Sq & R-Sq(adj) & $C p$ & $S$ & $\mathrm{~T}_{\mathrm{G} 2}$ & $\mathrm{~T}_{\mathrm{G} 17}$ & $\% \mathrm{Nb}$ & $\% \mathrm{~V}$ & $\% \mathrm{Mn}$ \\
\hline 1 & 77,6 & 71,2 & 3,6 & 1,5274 & & & & $X$ & \\
\hline 1 & 76,7 & 70,0 & 3,9 & 1,5583 & & & & & $X$ \\
\hline 2 & 80,6 & 70,9 & 4,6 & 1,5347 & & $x$ & & $X$ & \\
\hline 2 & 80,6 & 70,9 & 4,6 & 1,5364 & & $X$ & & & $X$ \\
\hline 3 & 82,6 & 68,6 & 5,9 & 1,5936 & $X$ & $X$ & & $X$ & \\
\hline 3 & 82,3 & 68,2 & 6,0 & 1,6058 & $x$ & & & $X$ & $X$ \\
\hline 4 & 90,3 & 78,1 & 5,3 & 1,3323 & $x$ & & $x$ & $X$ & $x$ \\
\hline 4 & 83,8 & 63,6 & 7,5 & 1,7160 & $X$ & $X$ & $X$ & $X$ & \\
\hline 5 & 91,1 & 73,4 & 7,0 & 1,4664 & $X$ & $X$ & $X$ & $X$ & $X$ \\
\hline
\end{tabular}

Após definidas quais as variáveis mais influentes no processo, foi realizado um modelamento matemático para definir uma equação que possibilitasse a previsão dos resultados das propriedades mecânicas. Este modelamento foi realizado através do software Minitab.

* Contribuição técnica ao $51^{\circ}$ Seminário de Laminação - Processos e Produtos Laminados e Revestidos, 28 a 31 de outubro de 2014, Foz do Iguaçu, PR, Brasil. 


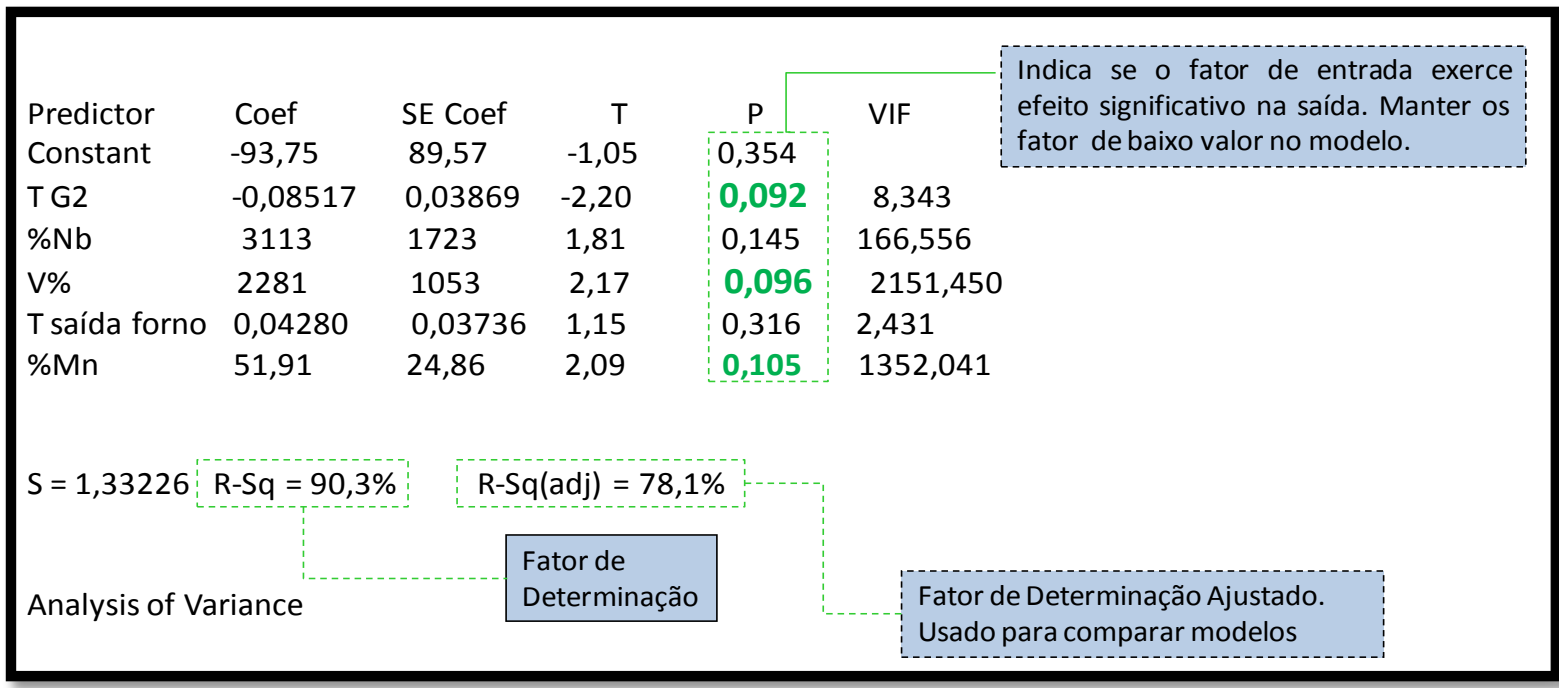

Figura 4 - Parâmetros retornados pelo software Minitab para análise e validação da equação.

Foram feitas várias simulações no software até encontrar uma equação matemática adequada.

A equação da regressão é:

Le $=-93,7-0,0852$ T G2 $+3113 \% N b+2281$ V\% + 0,0428 T saída forno + 51,9 \%Mn

Figura 5 - Equação otimizada obtida (experimento 2).

Com base na equação encontrada foram calculados os valores ótimos a serem utilizados para atingir os valores das propriedades mecânicas adequadas.

A equação mostrou que a influência do vanádio na determinação das propriedades mecânicas, mesmo que em pequeno teor é muito superior a influência do Nióbio.

Utilizando os parâmetros ótimo determinado pela equação acima eliminamos o nióbio e realizamos um novo teste, contendo apenas o vanádio. Para este experimento foi desenvolvido um novo aço (1622).

Tabela 4 - Tabela de composição química do aço 1622.

\begin{tabular}{|c|c|c|c|c|c|c|c|c|c|c|c|c|c|}
\hline \multirow{2}{*}{ Material } & \multicolumn{12}{|c|}{ Composição Química (\%) } & \multirow{2}{*}{$\begin{array}{l}\text { Requisitos } \\
\text { Adicionais }\end{array}$} \\
\hline & C & Si & Mn & $S$ & $\mathbf{P}$ & $\mathrm{Cu}$ & $\mathrm{Ni}$ & $\mathrm{Cr}$ & Sn & $\mathrm{Nb}$ & v & $\mathbf{N}$ & \\
\hline \multirow{2}{*}{ 1622-A } & 0,200 & 0,150 & 1,200 & - & - & - & - & - & - & - & 0,020 & - & \multirow{2}{*}{$\begin{array}{c}\text { ASTM A572 } \\
\text { G60 }\end{array}$} \\
\hline & 0,240 & 0,300 & 1,350 & 0,030 & 0,030 & 0,250 & 0,150 & 0,150 & 0,060 & - & 0,035 & 0,010 & \\
\hline
\end{tabular}

Após a realização do terceiro experimento a análise de melhor subgrupo de variáveis e o modelamento matemático foi refeito, visando validar os resultados obtidos. 


\section{LAMINAÇÃO

\begin{tabular}{|c|c|c|c|c|c|c|c|c|}
\hline Vars & R-Sq & $\begin{array}{c}\text { R- } \\
\text { Sq(adj) }\end{array}$ & $C p$ & s & $\operatorname{Mn}(\%)$ & $\mathrm{Nb}(\%)$ & $V(\%)$ & $\operatorname{TF}\left({ }^{\circ} \mathrm{C}\right)$ \\
\hline 1 & 43 & 39,2 & 3,1 & 1,0107 & $x$ & & & \\
\hline 1 & 35,7 & 31,5 & 6,9 & 1,073 & & & $x$ & \\
\hline 2 & 47,8 & 42,4 & 2,6 & 0,98409 & $x$ & & $x$ & \\
\hline 2 & 43,7 & $3 \underline{3}, \underline{8}$ & 4,7 & 1,022 & $\underline{x}$ & & & $\underline{x}$ \\
\hline 3 & $-48,6$. & 41,3 & $-4,1$ & 0,99311 & $\underline{x}$ & & $\underline{x}$ & $\underline{x}$ \\
\hline 3 & 48,3 & 40,9 & 4,3 & 0,99636 & $x$ & $x$ & $x$ & \\
\hline 4 & 48,8 & 39,4 & 6 & 1,0093 & $x$ & $x$ & $x$ & $x$ \\
\hline
\end{tabular}

Análise de Regressão: Le(kgf/mm²) versus $\mathrm{C}(\%) ; \mathrm{Mn}(\%)$; V(\%); TF Equação:

$\operatorname{Le}\left(\mathrm{kgf} / \mathrm{mm}^{2}\right)=21,5-18,6 \mathrm{C}(\%)+18,9 \mathrm{Mn}(\%)+113 \mathrm{~V}(\%)+0,00301 \mathrm{TF}$

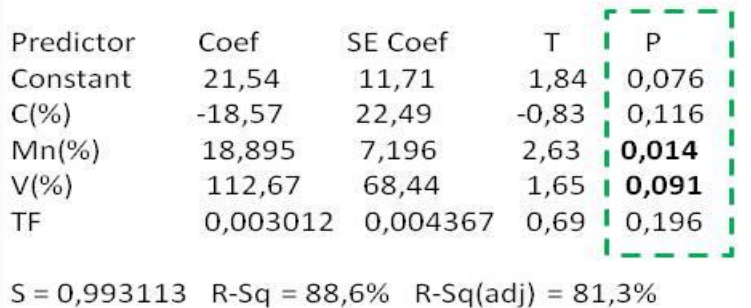

Figura 6 - Comprovação estatística da influência do Vanádio e geração de nova equação.

A equação final, encontrada através do modelamento matemático, foi validada e o erro médio encontrado foi de $1,85 \%$.

\section{RESULTADOS E DISCUSSÃO}

Os resultados obtidos nos experimentos estão representados a seguir no gráfico em formato de histograma, utilizado para determinar a capabilidade do processo em atender ao limite inferior (LSL) das propriedades mecânicas, conforme Norma ASTM A572 G60 de 2007.
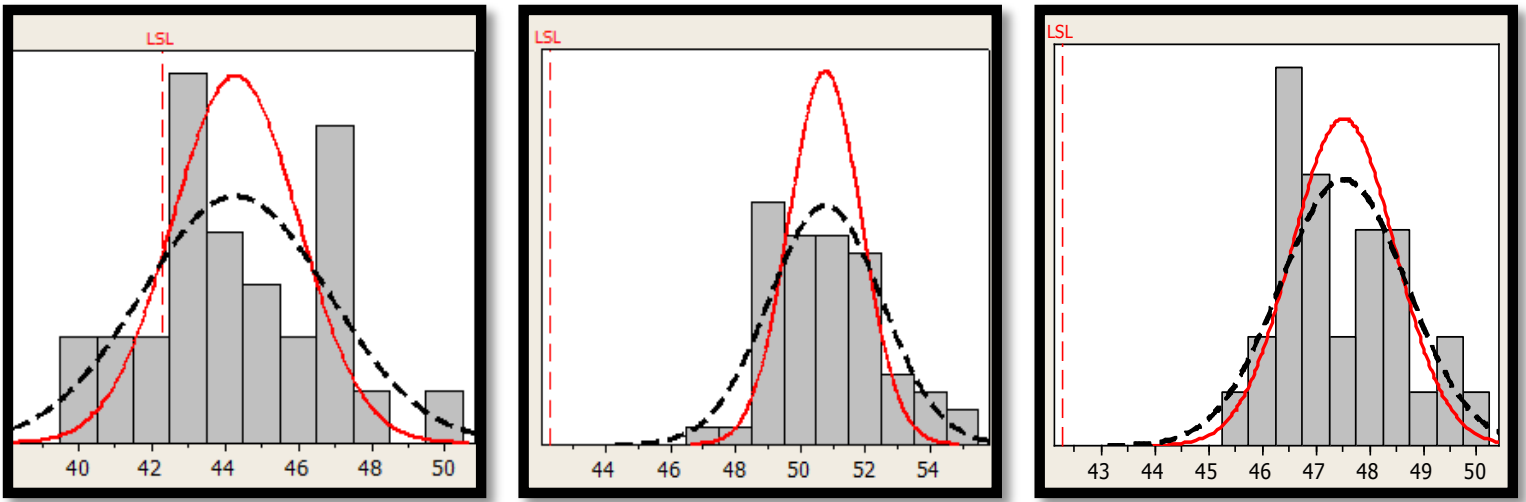

Figura 7 - Histograma de capabilidade dos experimentos 1, 2 e 3 respectivamente.

* Contribuição técnica ao $51^{\circ}$ Seminário de Laminação - Processos e Produtos Laminados e Revestidos, 28 a 31 de outubro de 2014, Foz do Iguaçu, PR, Brasil. 
Tabela 5 - Resultados dos experimentos.

\begin{tabular}{lccc}
\hline & Experimento 1 & Experimento 2 & Experimento 3 \\
\hline $\begin{array}{l}\text { Nível } \sigma \\
\text { (capabilidade) }\end{array}$ & 2,73 & 6 & 6 \\
$\begin{array}{l}\text { Probabilidade de não } \\
\text { atendimento (PNA) }\end{array}$ & $21,83 \%$ & $0 \%$ & $0 \%$ \\
Custo do aço R $\$$ /t & 98,89 & 103,29 & 73,26 \\
\hline
\end{tabular}

\section{CONCLUSÃo}

A iniciativa de utilizar um modelamento matemático que utilizasse parâmetros de processo e composição química do aço para prever as propriedades mecânicas de perfis ASTM A572 G60 foi um projeto pioneiro na unidade da Votorantim Siderurgia de Barra Mansa. As análises realizadas foram apropriadas ao objetivo do trabalho.

Após a realização de todos os experimentos e análises dos resultados, o processo mostrou-se estável em relação ao resultado das propriedades mecânicas do material com $100 \%$ de atendimento a especificação da norma.

A redução do custo da liga foi de $17 \%$ o que proporcional um ganho anual na ordem de $R \$ 450.00,00$

É importante destacar como um ganho relevante à satisfação do cliente e o aumento no número de vendas em $30 \%$ nestes perfis.

\section{Agradecimentos}

A equipe do projeto agradece a Votorantim Siderurgia, gerência de produtos e processos e a gerência de laminação a quente da unidade de Barra Mansa pelo apoio e incentivo aos experimentos realizados.

\section{REFERÊNCIAS}

1 ASTM A572 Grade 50 and 60 - Standard Specification for High-Strength Low-Alloy Columbium-Vanadium Structural Steel.American Society for Testing and Materials, 2007.

2 Bai DQ, et al. Effect of Deformation Parameters on the No- Recrystallization Temperature in Nb-Bearing Steels. Metallurgical Transactions A, 1993: 2151-2159.

3 Gorni AA, Cavalcanti CG. Modelamento Matemático das TemperaturasCríticas da Laminação Controlada através de Equações Empíricase Redes Neurais. LIII Congresso da Associação Brasileira de Metalurgia e Materiais, Belo Horizonte MG, 13 a 17 de Setembro de 1998.

4 Schiavo CP, Gonzalez BM, Santos AA, Marra KM. Influência dos Parâmetros de Solubilização Temperatura e Tempo de Encharque na Temperatura e Tempo de Encharque na $\mathrm{T}_{\mathrm{NR}}$ de um Aço Microligado Ao Nb, V, E TI. Tecnol. Metal. Mater. Miner., São Paulo, 2011; 8(1): 14-18.

5 Malarda B, Remyb B, Scottb C, Deschampsa A, Chênec J, Dieudonnéb T et al. Hydrogen trapping by VC precipitates and structural defects in a high strength Fe-Mn$\mathrm{C}$ steel studied by small-angle neutron scattering. Materials Science and Engineering $A$, 2012; 536: 110-116.

6 Campos MS. Desvendando o Minitab. Editora Qualitymark, 262, 2004.

7 Kiemele MJ, Schmidt SR. Basic Statistics - Tools for Continuous Improvement. Air Academy Pr; 4th edition June 1997. 